\title{
Modélisation des transferts gazeux dans le lit de semence : application au diagnostic des condi- tions d'hypoxie des semences de betterave su- crière (Beta vulgaris L.) pendant la germination. I. - Présentation du modèle
}

Guy RICHARD \& Jérôme GUÉRIF

I.N.R.A., Station de Science du Sol, Centre de Recherches d'Avignon, Domaine Saint-Paul, B.P.91, F84140 Montfavet

Mots clés additionnels : Aération, concentration en oxygène, coefficient de diffusion gazeuse, porosité libre à l'air, respiration microbienne du sol, croûte de battance.

Modeling gas transfer in seed beds : diagnosis of hypoxia conditions for sugar beet seed (Beta vulgaris L.) during germination. I. - Presentation of the model.

\begin{abstract}
A model based on gas diffusion laws (Fick's laws) in tilled layers of soil has been developed. It allows determination of the physical characteristics of seed beds and deeper layers which may inhibit the germination of sugar beet seed by a limitation of seed oxygen uptake. Within the profile, a possible crust, the seed bed and the ploughed layer have been distinguished. The gas transfer parameters (diffusion coefficient and transfer porosity) depend on the structural and water potential characteristics of each layer; their estimations are based on experimental relationships. Oxygen uptake by the seed is neglected. Soil microbial respiration depends on soil organic status and its variations with oxygen concentration and temperature have been taken into account. The model follows oxygen concentration in the seed bed and compares it with the oxygen concentrations, experimentally determined, which limit the germination of sugar beet seed.
\end{abstract}

Additional key words : Aeration, oxygen concentration, gas diffusion, air-filled porosity, soil microbial respiration, crust. 


\section{INTRODUCTION}

Certains accidents d'implantation des cultures semées sont, en raison des conditions climatiques et structurales au moment où ils interviennent (périodes humides, sols croûtés), attribués à une perturbation de la germination par une limitation de la fourniture d'oxygène à la semence. Cependant, on sait mal caractériser au champ les conditions d'alimentation en oxygène de la semence, et les observations réalisées le plus fréquemment à la levée ne permettent pas la distinction entre les problèmes liés à la phase de germination sensu stricto et ceux liés à la phase d'émergence.

Pour une culture comme la betterave sucrière, la phase d'implantation est déterminante vis-à-vis de la maîtrise de l'élaboration du rendement. La tendance à des semis de plus en plus précoces dans des zones pédoclimatiques à fort risque de dégradation structurale fait que le problème de l'apparition de conditions hypoxiques dans le lit semence se pose particulièrement pour cette culture. On se propose donc, pour la betterave sucrière, de déterminer les caractéristiques du lit de semence susceptibles de limiter la consommation d'cxygène de la semence et de perturber sa germination, afin de mettre au point des moyens de diagnostic des conditions d'hypoxie dans le lit de semence.

A partir d'une modélisation des transferts gazeux, on cherchera à estimer l'influence des caractéristiques d'état du lit de semence sur les profils de concentration en oxygène dans celui-ci et sur leurs cinétiques. La validation d'un tel modèle est difficile. On ne dispose pas de moyen de mesure fiable et opérationnel de la teneur en oxygène in situ. On pouvait espérer utiliser un indicateur biologique, mesuré au niveau de la semence, et corrélé à la concentration en oxygène autour de la semence. La charge énergétique (comme définie par Pradet \& RAYmond, 1983) dépend de la vitesse de respiration et de l'activité des processus fermentaires de la semence, qui varient avec la concentration en oxygène. Dans le cas de la semence de betterave sucrière, la charge énergétique ne s'est pas révélée être suffisamment sensible à la concentration en oxygène pour être utilisée comme un marqueur de celle-ci (RICHARD 1988). On ne cherchera donc pas à valider le modèle de manière physique. Mais, à partir de relations déterminées expérimentalement entre la concentration en oxygène au niveau de la semence de betterave sucrière, sa consommation d'oxygène et son comportement en germination, on définira les conditions considérées comme critiques vis-à-vis de la germination. En confrontant les profils de concentration dans le lit de semence obtenus par simulation aux concentrations dites critiques, nous pourrons alors mettre en évidence les situations susceptibles d'entraîner des risques de perturbation de la germination.

Dans ce premier article, on présente les bases physiques de la modélisation des transferts gazeux dans le sol pour une étude des conditions d'hypoxie dans le lit de semence, et le mode d'estimation des paramètres de transfert. On se donne enfin une gamme probable d'états du profil à partir desquels on calculera l'évolution de la concentration en oxygène dans le lit de semence. Les résultats des simulations sont présentés dans un second article (RICHARD \& GUÉRIF, 1988).

\section{BASES PHYSIQUES DE LA MODÉLISATION DES TRANSFERTS GAZEUX}

La liste des symboles utilisés est présentée dans le tableau 1.

TABLEAU 1

Symboles utilisés.

Symbols used.

\begin{tabular}{|c|c|}
\hline $\mathrm{x}$ & profondeur \\
\hline$r$ & rayon \\
\hline $\mathrm{t}$ & temps \\
\hline $\mathrm{T}$ & température \\
\hline $\mathrm{C}$ & $\begin{array}{l}\text { concentration en } \\
\text { oxygène }\end{array}$ \\
\hline $\mathbf{P}$ & $\begin{array}{l}\text { fonction puits à } \\
\text { oxygène }\end{array}$ \\
\hline q & flux d'oxygène \\
\hline $\mathrm{D}$ & $\begin{array}{l}\text { coefficient de } \\
\text { diffusion apparent de l'oxygène dans le sol }\end{array}$ \\
\hline $\mathrm{D} / \mathrm{D}_{0}$ & $\begin{array}{l}\text { coefficient de } \\
\text { diffusion relatif du sol }\end{array}$ \\
\hline $\mathrm{N}$ & $\begin{array}{l}\text { porosité de } \\
\text { transfert }\end{array}$ \\
\hline $\mathrm{N}_{\mathrm{a}}$ & $\begin{array}{l}\text { porosité libre à } \\
\text { l'air }\end{array}$ \\
\hline $\mathrm{e}$ & $\begin{array}{l}\text { épaisseur de la } \\
\text { croûte }\end{array}$ \\
\hline $\mathrm{p}$ & profondeur du lit de semence \\
\hline ps & $\begin{array}{l}\text { profondeur de } \\
\text { semis }\end{array}$ \\
\hline $\mathrm{L}$ & profondeur de la couche labourée \\
\hline 2. $\mathrm{R}$ & maille de fissuration \\
\hline Is & limite inférieure du profil \\
\hline
\end{tabular}

\section{A. Présentation des lois physiques}

Deux processus physiques sont responsables des transferts gazeux: le mass-flow et la diffusion. Le processus dominant dans le sol étant la diffusion gazeuse, on négligera les transferts par mass-flow : on suppose que l'atmosphère du sol est à la pression atmosphérique et que les gradients thermiques sont faibles.

Les transferts par diffusion sont régis par la loi de Fick qui, sous sa forme monodimensionnelle, s'écrit de la façon suivante :

$$
q_{x}=-D_{x} \cdot \frac{\partial C}{\partial x}
$$

$(\mathrm{Ox}):$ axe des transferts.

$\mathrm{q}_{\mathrm{x}}$ : flux de gaz selon $\mathrm{Ox}$, rapporté à une section de sol $\left(\mathrm{kg} \mathrm{m}^{-2} \mathrm{~s}^{-1}\right)$.

$D_{x}$ : coefficient de diffusion selon $O x\left(\mathrm{~m}^{2} \cdot \mathrm{s}^{-1}\right) \cdot D_{x}$ représente le coefficient de diffusion apparent du gaz dans le sol considéré.

$\mathrm{C}$ : concentration du gaz rapportée au volume d'air $\left(\mathrm{kg} \cdot \mathrm{m}^{-3}\right)$.

La loi de Fick est une loi strictement empirique, établie à partir d'études sur les transferts de solutés. Des travaux montrant son accord avec le processus de diffusion en milieu poreux ont permis de l'étendre aux transferts de gaz par diffusion (JAYNES \& ROGOWSKI, 1983). 
Par ailleurs, la condition de conservation de masse conduit à l'équation suivante :

$$
N_{x} \cdot \frac{\partial C}{\partial t}=-\frac{\partial q_{x}}{\partial x}-P(x, C, t)
$$

$\mathrm{x}$ : porosité de transfert $\left(\mathrm{m}^{3} \mathrm{~m}^{-3}\right)$.

$t$ : temps (s).

$\mathrm{P}(\mathrm{x}, \mathrm{C}, \mathrm{t})$ : fonction puits $(\mathrm{P} \geqslant 0)$ rapportée au volume de sol $\left(\mathrm{kg} \cdot \mathrm{m}^{3} \cdot \mathrm{s}^{-1}\right)$.

Si le milieu n'est pas saturé en eau, $\mathrm{C}$ représente la concentration du gaz par rapport au volume d'air et $\mathrm{N}$ la porosité libre à l'air accessible au gaz.

Si le milieu est saturé en eau, $\mathrm{C}$ sera considérée comme la concentration équivalente à celle du gaz dans l'air qui serait en équilibre avec la phase liquide du volume élémentaire de sol. $\mathrm{N}$ représente alors la porosité totale accessible au gaz, multipliée par le coefficient de solubilité du gaz considéré.

Pour la commodité d'expression des résultats, la concentration en oxygène et la fonction puits seront exprimées respectivement en p. 100 et en $\mathrm{mm}^{3}$ d'oxygène $\cdot \mathrm{h}^{-1} \cdot \mathrm{cm}^{-3}$ de sol dans la suite du texte.

En coordonnées axi-cylindriques, compte tenu d'une symétrie radiale, l'équation (2) s'écrit (in GLINSKI \& STEPNIEWSKI, 1985) :

$$
\begin{aligned}
N_{r, x} \cdot \frac{\partial C}{\partial t}= & -\frac{1}{r} \cdot \frac{\partial\left(r \cdot q_{r}\right)}{\partial r}-\frac{\partial q_{x}}{\partial x} \\
& -P(r, x, C, t)
\end{aligned}
$$

(Or) représentant l'axe radial.

\section{B. Présentation du système étudié}

\section{Origines des variations de concentration en oxygène; nature des fonctions puits prises en compte}

Les transferts d'oxygène par diffusion dans le sol résultent d'un gradient de concentration en oxygène lié à une consommation d'oxygène en au moins un point du système. Cette fonction puits à oxygène correspond soit à la consommation d'oxygène de la semence, soit à celle des microorganismes du sol. L'ordre de grandeur et les échelles d'espace concernées dans le profil par ces 2 fonctions puits sont très différents :

- la fonction puits « semence" est de moins de 5 $\mathrm{mm}^{3} \cdot \mathrm{h}^{-1}$ (RICHARD 1988) et elle est locale,

- la fonction puits «microorganismes » est répartie sur l'ensemble du profil et, mesurée à la surface du sol, elle représente en moyenne une consommation d'oxygène de $30 \mathrm{~mm}^{3} \cdot \mathrm{h}^{-1} \cdot \mathrm{cm}^{2}$ (CURRIE, 1962).

La concentration en oxygène au niveau de la semence résulte donc :

- d'une part, des capacités de transfert globales du lit de semence par rapport à la fonction puits à oxygène «microorganismes » de l'ensemble du profil ; la fonction puits « semence » est alors négligeable;

- d'autre part, des conditions locales de diffusion de l'oxygène à l'interface semence/sol (contact «terresemence ", présence d'un film d'eau autour de la semence, présence d'un lissage lié au semoir) et de la fonction puits « semence".
Chacune des 2 causes des variations de la concentration en oxygène autour de la semence nécessite une étude spécifique. Cet article ne concerne que les capacités globales de transfert d'oxygène du lit de semence. Le modèle ainsi élaboré devra permettre de fixer les conditions aux limites d'un autre modèle de transfert de l'oxygène qui ne concernera que l'environnement immédiat de la semence.

On ne prend donc en compte que la fonction puits d'origine microbienne. Le système étudié est alors réduit uniquement à un volume de sol présentant une activité microbienne répartie sur l'ensemble du profil (lit de semence et couches sous-jacentes).

Différents travaux ont déjà été consacrés à l'étude des conséquences de l'activité biologique sur la concentration en oxygène dans le profil à partir d'une modélisation des lois de diffusion gazeuse (in GLINSKI \& STEPNiEWSKI, 1985). Cependant aucun ne concerne spécifiquement le lit de semence et la phase de germination. Le lit de semence se caractérise par sa position à proximité de l'interface sol/atmosphère : il est proche de la source d'oxygène qu'est l'atmosphère, mais il est aussi directement soumis aux agressions climatiques. Une croûte de battance peut alors se former, fortement limitante vis-à-vis de la diffusion gazeuse et souvent de structure hétérogène; sa prise en compte nécessite de travailler en coordonnées tridimensionnelles.

\section{Caractérisation $d u$ profil de sol; définition des systèmes étudiés, conditions aux limites}

Compte tenu du risque élevé de dégradation structurale en surface au moment des semis, le sol étant nu à cette période, on peut distinguer a priori 4 couches au sein du profil, comme indiquées figure 1. En dehors de la croûte de battance, chaque couche est supposée homogène vis-à-vis de la porosité de transfert et de la fonction puits, et isotrope vis-à-vis du coefficient de diffusion.

En ce qui concerne la croûte de battance, on considérera que :

- soit elle est continue, homogène et isotrope ; le système se réduit alors à un profil monodimensionnel,

-.- soit elle est fissurée, homogène et isotrope entre les fissures; le système sera alors assimilé à un cylindre (figure 2) de diamètre équivalent à la maille de fissuration, et on considérera une symétrie radiale.

Comme conditions aux limites, on prendra :

- en surface, une condition de concentration constante : $\mathrm{C}(\mathrm{x}=0)=\mathrm{C}_{0}$,

- en profondeur, faute de données précises, on négligera l'activité biologique du sous-sol et la réserve d'oxygène qu'il représente, et l'on imposera une condition de flux nul à la limite de la couche labourée, soit : $\mathrm{q}_{\mathrm{x}}(\mathrm{x}=\mathrm{L})=0$.

Dans le cas d'un profil avec une croûte fissurée, on prendra comme conditions aux limites:

- sur la face latérale du cylindre :

- une condition de concentration constante sur l'épaisseur de la croûte :

$\mathrm{C}(\mathrm{r}=\mathrm{R}, 0 \leqslant \mathrm{x} \leqslant \mathrm{e})=\mathrm{C}_{0}$,

- une condition de flux nul sur le reste de la face par construction : $\mathrm{q}_{\mathrm{r}}(\mathrm{r}=\mathrm{R}, \mathrm{x}>\mathrm{e})=0$, 


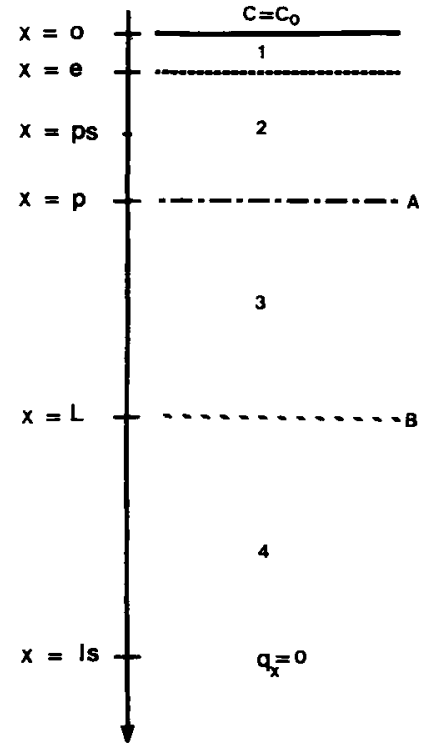

Figure 1

Présentation de la structure multicouche du profil.

1) croûte de battance,

2) lit de semence.

3) couche labourée non reprise

4) sous-sol,

A) limite du travail du sol avant semis.

B) limite du labour.

« $x$ » indique la profondeur de chaque couche.

Presentation of the multilayer structure of the profile.

1) crust,

2) seed bed,

3) ploughed layer

4) sub-soil,

A) seed bed-ploughed layer interface,

B) ploughing depth limit,

" $x$ " indicates the depth of each layer.

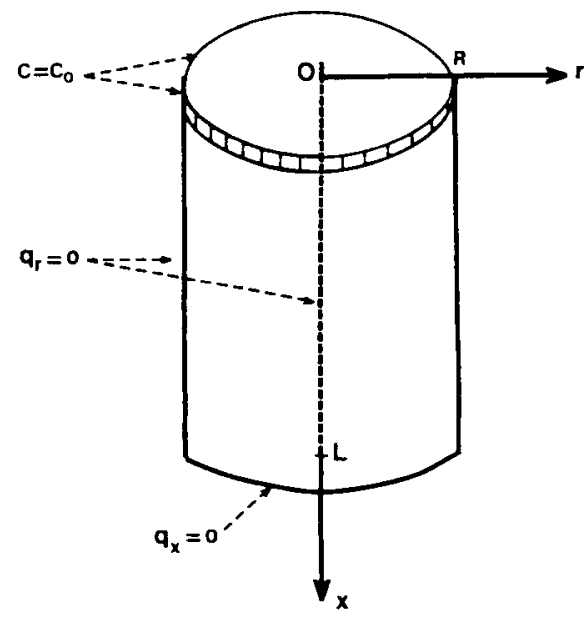

Figure 2

Géométrie du système en coordonnées axi-cylindriques (Profil avec une croûte fissurée). croûte de battance.

Geometry of the system in axi-cylindric coordinates (profile with a cracked crust). crust.

- sur l'axe du cylindre, une condition de flux nul par symétrie : $\mathrm{q}_{\mathrm{r}}(\mathrm{r}=0)=0$.
Comme conditions initiales, sauf précisions dans le texte, on prendra une concentration uniforme égale à celle de l'atmosphère en tous points du système.

\section{ESTIMATION DES PARAMÈTRES DU MODĖLE}

\section{A. Estimation du coefficient de diffusion et de la porosité de transfert}

1. Problèmes posés par l'estimation du coefficient de diffusion et de la porosité de transfert

On ne dispose pas de modèle de prévision (déterministe ou empirique) de ces 2 paramètres en fonction de caractéristiques du milieu de transfert, et la mesure de ces paramètres in situ est difficile. On estimera les valeurs de ces paramètres en recherchant des relations empiriques qui les relient à des variables d'états accessibles (potentiel de l'eau, indice des vides), pour des cas-types d'états physiques correspondant aux situations extrêmes risquant d'être rencontrées lors des semis.

On peut caractériser l'espace poral du profil en distinguant l'espace poral textural essentiellement défini par l'assemblage des constituants du sol (STENGEL, 1979) et son complémentaire, l'espace poral structural, principalement dû au travail du sol et aux facteurs climatiques. Ces 2 sous-ensembles sont constitués de pores de nature et de taille très différentes dans lesquelles la vitesse de diffusion des gaz est elle aussi très différente : le coefficient de diffusion d'un gaz devient dépendant de la taille des pores à partir de diamètres inférieurs à $2 \mu \mathrm{m}$ (CLIFFORD \& HILLEL, 1986). L'utilisation de relations empiriques entre des variables d'état du sol et le coefficient de diffusion apparent d'un gaz suppose donc la connaissance de la répartition du volume de la phase gazeuse entre l'espace poral textural et structural.

L'apparition de conditions risquant de limiter la fourniture d'oxygène à la semence étant liée à des conditions pluvieuses, on peut supposer la porosité d'origine texturale saturée (sauf pour des textures grossières). Il s'agit donc d'établir des relations empiriques entre des variables d'état du sol et le coefficient de diffusion apparent de l'oxygène pour des sols où la porosité libre à l'air est uniquement d'origine structurale. Des mesures de coefficient de diffusion ont été réalisées dans ces conditions.

En ce qui concerne la porosité de transfert, comme dans toute la littérature, elle sera assimilée en milieu non saturé à la porosité libre à l'air $\left(\mathrm{N}_{\mathrm{a}}\right)$ : on néglige la fraction du volume gazeux qui peut ne pas être connectée avec le reste de la porosité libre à l'air; en milieu saturé, la porosité de transfert sera assimilée à la porosité totale multipliée par le coefficient de solubilité de l'oxygène.

\section{Mesure du coefficient de diffusion}

Le coefficient de diffusion $\mathrm{D}$ est obtenu selon le protocole de BALL et al. (1981) basé sur la diffusion d'un 
traceur radioactif $\left({ }^{85} \mathrm{Kr}\right)$ et par la méthode d'estimation de BRUCKLER et al. (1988) sur des éprouvettes de sol de $7 \mathrm{~cm}$ de diamètre et de $2,4 \mathrm{~cm}$ d'épaisseur. On estime alors le coefficient de diffusion apparent du Krypton dans l'éprouvette de sol. Le coefficient de diffusion relatif de l'éprouvette, égal au rapport $D / D_{0}$ avec $D_{0}$ coefficient de diffusion du gaz dans l'air libre, étant identique en première approximation quel que soit le gaz considéré, on peut calculer le coefficient de diffusion apparent de l'oxygène dans l'éprouvette de sol $\left(\mathrm{D}_{0}=0,2 \cdot 10^{-4} \mathrm{~m}^{2} \cdot \mathrm{s}^{-1}\right.$ pour l'oxygène à $20^{\circ} \mathrm{C}$ (in GLINSKI \& STEPNIEWSKI, 1985)).

Les éprouvettes de sol sont obtenues par compactage standard, dans une cellule de volume constant, de quantités d'agrégats calculées pour obtenir la gamme de porosité de transfert visée. Les agrégats sont obtenus par un tamisage à sec (de diamètre 1 à $2 \mathrm{~mm}$ ou 2 à $3 \mathrm{~mm}$ ). Amenés préalablement à saturation (potentiel hydrique de $-0,3 \mathrm{kPa}$ ) sous vide, ils sont ensuite desséchés sous gel de silice jusqu'à une humidité choisie dans le domaine de saturation, déterminée à partir d'une courbe de retrait établie à partir d'agrégats de 2 à $3 \mathrm{~mm}$ de diamètre. L'éprouvette de sol est caractérisée par un indice des vides structuraux résultant et une humidité, et par une porosité structurale libre à l'air ; au moment de sa fabrication, le taux de saturation de la porosité structurale est nul (à un potentiel de $-20 \mathrm{kPa}$ ). Les variations du taux de saturation de la porosité structurale sont obtenues à partir d'éprouvettes de sol fabriquées comme décrit précédemment, amenées à saturation totale (potentiel nul) puis mises à l'équilibre sur une table de tension à de forts potentiels $(-1$,

$-2 \mathrm{kPa}$ ). Le sol utilisé est un limon argileux à $26 \mathrm{p} .100$ d'argile.

Les résultats sont présentés sur la figure 3 ; ils sont conformes aux données de la littérature obtenues dans des conditions similaires (PAPENDICK \& RUNKLES, 1965 ; STEPNIEWSKI, 1981 ; CURRIE, 1984). L'intérêt du protocole adopté est la maîtrise de la répartition de l'eau

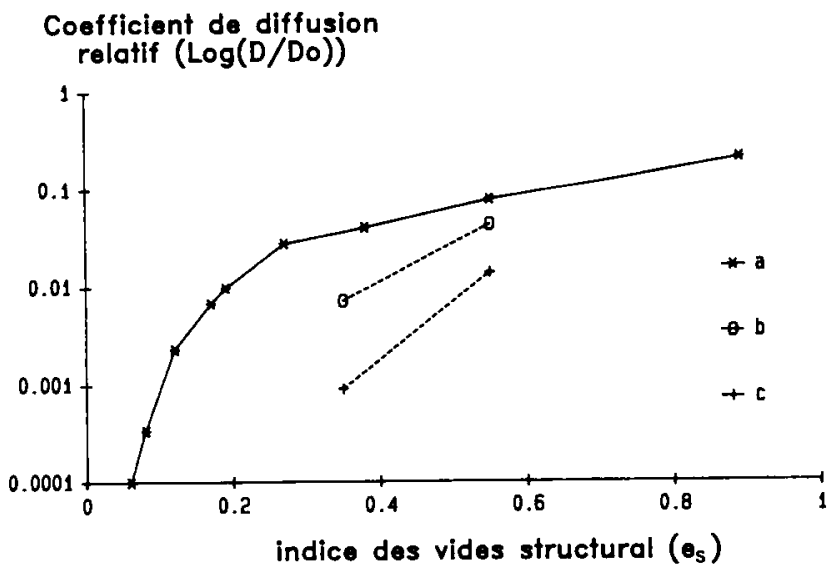

Figure 3

Coefficient de diffusion relatif $\left(D / D_{0}\right)$ en fonction de l'indice des vides structuraux $\left(e_{s}\right)$, du potentiel de l'eau (î) et du diamètre des agrégats (d).

Relationships between relative diffusion coefficient $\left(D / D_{0}\right)$, structural void ratio $\left(e_{s}\right)$, water potential $(I)$ and aggregate diameter $(d)$.

a) $\hat{I}=-20 \mathrm{kPa} \quad d=2-3 \mathrm{~mm}$,

b) $\hat{I}=-1 \mathrm{kPa} \quad d=2-3 \mathrm{~mm}$,

c) $\hat{I}=-1 \mathrm{kPa} \quad d=1-2 \mathrm{~mm}$. entre les volumes textural et structural assimilables en première approximation au volume intra et interagrégats. Le coefficient de diffusion est indépendant de la taille des pores pour des diamètres supérieurs à $2 \mu \mathrm{m}$ (Clifford \& Hillel, 1986); vu la taille des pores d'origine structurale, il est donc indépendant de la taille des agrégats, sous réserve que les arrangements alors obtenus soient comparables. On admet donc que la courbe a (taux de saturation de la porosité structurale nul) est généralisable à n'importe quel type de matériau pour lequel la porosité d'origine texturale est saturée. Pour des taux de saturation de la porosité structurale non nuls c'est-à-dire à de forts potentiels de l'eau, le coefficient de diffusion est dépendant de la taille des agrégats utilisés (courbes $\mathbf{b}$ et $\mathbf{c}$ ); l'extrapolation des relations représentées par les courbes $\mathbf{b}$ et $\mathbf{c}$ nécessite alors la connaissance de la gamme de variation de taille des agrégats présents dans le milieu.

\section{B. Estimation de la fonction puits à oxygène d'origine microbienne}

\section{Rôle du statut organique}

Pour des sols cultivés à teneur en carbone organique voisine de 1 p. 100 , où il n'y a pas eu incorporation récente de résidus de récolte ou d'amendements organiques, la fonction puits à oxygène, mesurée en laboratoire, varie entre 0,1 et $2,5 \mathrm{~mm}^{3} \cdot \mathrm{h}^{-1} \cdot \mathrm{cm}^{3}$ pour des températures de 20 à $27^{\circ} \mathrm{C}$ (BROWN et al., 1965; BRIDGE \& Rixon, 1976 ; in GlinSKI \& STEPNIEWSKI, 1985 ; BoONE et al., 1986).

Après apport de 1 à 2 p. 100 de résidus de récolte, la fonction puits augmente en quelques jours jusqu'à des valeurs de 10 à $20 \mathrm{~mm}^{3} \cdot \mathrm{h}^{-1} \cdot \mathrm{cm}^{-3}$ (pour des températures de 20 à $27^{\circ} \mathrm{C}$ ), puis diminue progressivement dans les mois qui suivent jusqu'à atteindre son niveau de base antérieur (LYDA \& ROBINSON, 1969 ; GUÉRIF, 1986).

\section{Effets de la température et de l'humidité}

Aucune loi ne rend bien compte des variations de la consommation d'oxygène des microorganismes du sol en fonction de la température qui modifie à la fois la population microbienne et sa cinétique respiratoire (GLINSKI \& StePNIEWSKI, 1985). Une des lois néanmoins utilisée est la loi empirique de VAN'T OFF ou loi du $Q_{10}$ qui se présente sous la forme suivante :

$$
\log \left(\mathrm{P}_{1} / \mathrm{P}_{2}\right)=\left(\mathrm{T}_{1}-\mathrm{T}_{2}\right) \cdot \log \left(\mathrm{Q}_{10}\right) / 10,
$$

$\mathrm{T}$ étant la température.

$\mathrm{Q}_{10}$, qui représente le taux d'accroissement de la vitesse de la réaction considérée pour une variation de $10^{\circ} \mathrm{C}$, varie en moyenne entre 2 et 3 en fonction du type de sol (Ross \& CAIRNS, 1978; HowARD \& HowARD, 1979). Cependant, $Q_{10}$ n'est pas constant avec la température et varie entre 1,5 et 3 pour des températures variant entre 10 et $25^{\circ} \mathrm{C}$, et entre 3 et 5 pour des températures variant entre 5 et $15^{\circ} \mathrm{C}$ (Ross \& CAIRNS, 1978).

L'humidité sera supposée n'être jamais suffisamment faible pour réduire l'activité microbienne.

\section{Effet de la concentration en oxygène}

La consommation d'oxygène par les microorganismes diminue à partir d'une concentration en oxygène de 
10 p. 100 (Grable \& Siemer, 1968) et elle est réduite de moitié pour une concentration en oxygène de l'ordre de 2 p. 100 (in GLINSKI \& STEPNIEWSKI, 1985). A partir des données de PAAR \& REuszer (1959) et de celles contenues dans ICW REPORT (1975) présentées sur la figure 4, il apparaît que l'on peut traduire la variation de la consommation d'oxygène en fonction de la concentration en oxygène par une courbe de type MichaelisMenten, comme c'est le cas au niveau cellulaire (GREENwOOD, 1961), avec un coefficient $\mathrm{K}_{\mathrm{m}}$ de $2,5 \cdot 10^{-2}$. On obtient donc la relation suivante :

$$
\mathrm{P}(\mathrm{C})=\mathrm{P}_{\max } /\left(1+\mathrm{K}_{\mathrm{m}} / \mathrm{C}\right)
$$

avec

$\mathrm{K}_{\mathrm{m}}=2,5 \cdot 10^{-2}$ et $\mathrm{P}_{\max }=\mathrm{P}_{21 \%} \cdot\left(1+\mathrm{K}_{\mathrm{m}} /\left(21 \cdot 10^{-2}\right)\right)(5)$.

Nous considérerons cette relation comme valide quels que soient les états structural, hydrique et organique, du milieu et la température.

\section{GAMMES DE VARIATION DES PARAMĖTRES RETENUS POUR LA SIMULATION}

Le tableau 2 présente la gamme de variation de l'etat structural caractérisé par l'indice des vides structuraux, la profondeur, le coefficient de diffusion relatif et la porosité de transfert de la croûte de battance, du lit de semence et de la couche labourée sous-jacente.

D'après le modèle de dégradation structurale des couches superficielles de BoIfFIN (1984), la croûte de battance a une structure continue, et on peut considérer qu'elle est initialement saturée. Elle ne se désature ou ne se fissure qu'après une période de dessèchement. On ne dispose pas de référence pour une croûte désaturée; du

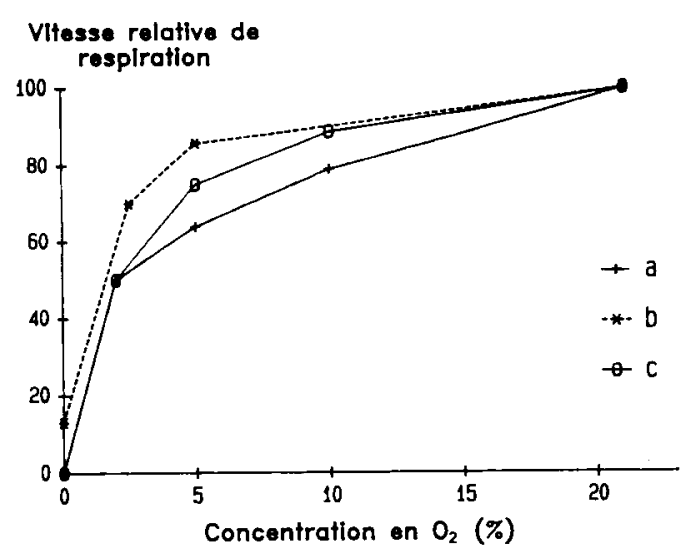

Figure 4

Variation de la vitesse relative de respiration d'origine microbienne du sol en fonction de la concentration en oxygène.

a) d'après ICW REPORT (1975) (en p. 100 de la consommation horaire d'oxygène à 21 p. 100 d'oxygène).

b) d'aprés PARR \& REUSZER (1959) (en p. 100 de la production cumulée de $\mathrm{CO}_{2}$ à 21 p. 100 d'oxygène).

c) d'après la relation (5).

Relationship between soil microbial respiration and oxygen concentration

a) according to ICW REPORT (1975) (in \% of oxygen uptake for $1 \mathrm{~h}$ at $21 \%$ of oxygen).

b) according to PARR \& REUSZER (1959) (in \% of $\mathrm{CO}_{2}$ cumulated production at $21 \%$ of oxygen).

c) according to relation (5)

fait de sa structure continue, on a considéré des coefficients de diffusion relatifs de la croûte faibles, variables entre $10^{5}$ et $10^{3}$.

L'indice des vides structuraux minimum dans le lit de

TABLEAU 2

Gammes de variation de l'état structural et des paramètres dé transferts de chaque couche de profil de sol comme définie sur la figure 1. Variation scales of structural characteristics cind diffusion parameters for each layer as defined on figure 1.

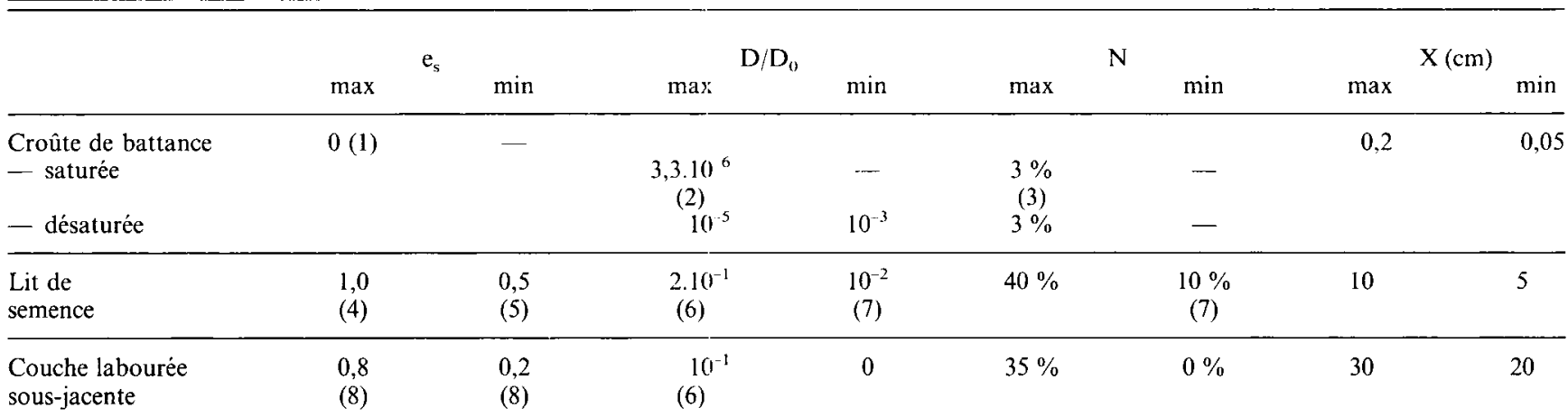

\footnotetext{
(1) BOIFFIN, 1984.

(2) Glinski \& Stepniewski (1985).

(3) Porosité totale de $50 \%$ et coefficient de solubilité de l'oxygène de $3,3 \%$ à $20^{\circ} \mathrm{C}$ (GLINSKI \& STEPNIEWSK1, 1985).

(4) BolfFin, 1984 ; Bouaziz, 1987.

(5) DeXTER et al., 1983 ; BOIFFin, 1984.

(6) D'après la figure 3.

(7) Valeurs pour un potentiel de $-1 \mathrm{kPa}$, des agrégats de 1 à $2 \mathrm{~mm}$ de diamètre et pour l'indice des vides structural le plus faible (fig. 3 )

(8) BOONe et al., 1986; Stengel, 1986 ; BOUAZIZ, 1987.

(3) Total porosity of $50 \%$ and oxygen solubility coefficient of $3,3 \%$ at $20^{\circ} \mathrm{C}$.

(6) According to figure 3.

(7) Values for a potential of $-1 \mathrm{kPa}$, aggregates calibrated between $1-2 \mathrm{~mm}$ and for the lower structural void ratio (fig. 3).
} 
semence a été estimé, d'après BoIFFIN (1984), comme résultant de l'évolution d'un lit de semence ayant un indice des vides structuraux initial de 0,8 et ayant subi une quantité totale de pluies cumulées de $50 \mathrm{~mm}$. Le potentiel de $-1 \mathrm{kPa}$ représente le potentiel maximal retenu; on n'a pas pris en compte le cas où le lit de semence est totalement saturé.

Puisqu'entre le coefficient de diffusion relatif et la porosité de transfert il n'y a pas une relation unique (pour des taux de saturation de la porosité structurale non nuls), il sera nécessaire de prendre, pour un même coefficient de diffusion relatif, des porosités libres à l'air différentes, et vice-versa.

On considérera des fonctions puits à oxygène potentielles (température de $20^{\circ} \mathrm{C}$, concentration en oxygène de $21 \mathrm{p}$. 100) de moins de $3 \mathrm{~mm}^{3} \cdot \mathrm{h}^{1} \cdot \mathrm{cm}^{3}$ en l'absence de résidus de récolte et de 5 à $10 \mathrm{~mm}^{3} \cdot \mathrm{h}^{-1} \cdot \mathrm{cm}^{3}$ en présence de résidus de récolte, dans le lit de semence et dans la couche labourée sous-jacente.

La température variera entre 5 et $10^{\circ} \mathrm{C}$ pour la température minimale et entre 10 et $20^{\circ} \mathrm{C}$ pour la température maximale.

On ne prendra en compte, ni les variations du coefficient de diffusion en fonction de la température, négligeables face à celles de la fonction puits, ni les variations du coefficient de diffusion en fonction de la pression atmosphérique.

\section{DÉFINITION DES SITUATIONS CRITIQUES}

En fonction de la concentration en oxygène, la vitesse de consommation d'oxygène par la semence varie. Si l'on pouvait définir, comme pour l'eau (BRUCKLER, 1983), les besoins en oxygène de la semence pour que la germination ait lieu en terme de quantité d'oxygène à absorber, alors, à partir de la relation entre la concentration en oxygène et la vitesse de consommation d'oxygène, on calculerait les flux d'oxygène pénétrant dans la semence, la quantité cumulée d'oxygène absorbée et ainsi, on pourrait prévoir le temps de germination. Mais, ne pouvant traduire le stade «germination » par une quantité donnée d'oxygène ( RICHARD 1988), on ne peut définir les situations critiques vis-à-vis de la germination qu'à partir de relations de références entre la concentration en oxygène au niveau de la semence et le comportement en germination.

La figure 5 présente les cinétiques de germination pour différentes concentrations en oxygène obtenues selon le protocole de RICHARD (1988). Il apparaît que la concentration devient limitante aux alentours de 10 p. 100 : on observe alors une augmentation du temps de germination et une légère diminution du taux de germination. A 1 p. 100 la germination est pratiquement inhibée. Ces résultats sont en accord avec ceux de PERRY \& HARRISON (1974) sur un autre lot de semences.

Les valeurs de références de comportement en germination, fixées à 10 p. 100 et 1 p. 100, ont été déterminées expérimentalement en maintenant une concentration en oxygène et une température constantes au cours du temps. Or dans un lit de semence, la concentration en oxygène initialement de 21 p. 100 au niveau de la

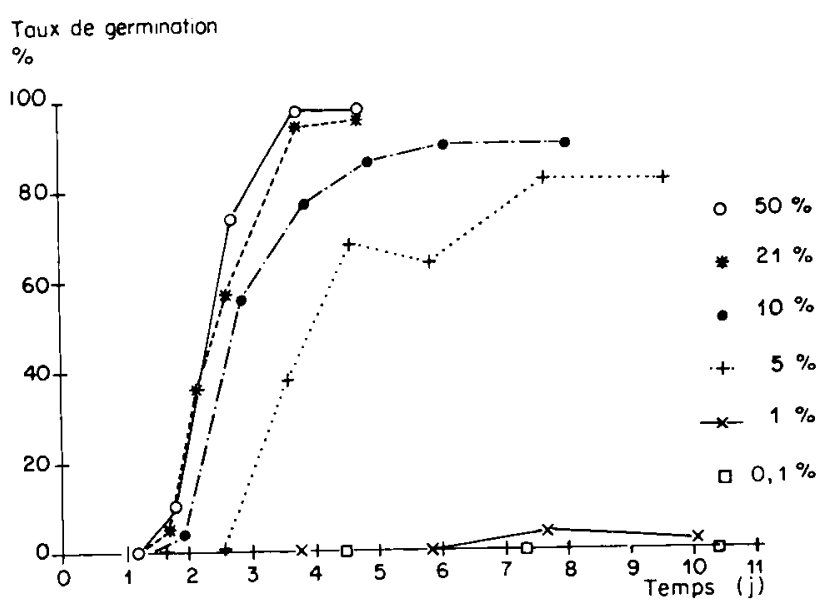

Figure 5

Germination des semences de betterave sucrière en fonction de la concentration en oxygène, $\dot{a} 20^{\circ} \mathrm{C}$ dans des conditions optimales d'imbibition (RICHARD, 1988).

Sugar beet seed germination according to oxygen concentration at $20^{\circ} \mathrm{C}$ in optimal water conditions (RICHARD, 1988).

semence va, au cours du temps, varier en fonction de l'état initial du lit de semence et des conditions climatiques. Mais, on ne connaît pas a priori les conséquences, sur le comportement ultérieur de la semence, d'un séjour momentané en hypoxie. Ces conséquences doivent en plus dépendre de la température, comme c'est le cas pour les valeurs de référence de comportement en germination qui sont plus faibles à basses températures (JUNTILlA, 1974). On ne peut pas envisager de déterminer expérimentalement ces conséquences de manière exhaustive, et on ne peut donc pas définir avec précision la gamme des situations critiques.

Cependant, la valeur critique de 10 p. 100 de concentration en oxygène peut être considérée comme un seuil maximal puisqu'elle a été déterminée à $20^{\circ} \mathrm{C}$. On considérera qu'à partir du moment où la concentration en oxygène devient inférieure à $10 \mathrm{p}$. 100 , le comportement en germination de la semence de betterave sucrière risque d'être perturbé (ralentissement de la vitesse de germination); on surestime ainsi les risques d'apparition de conditions limitantes dans le lit de semence. Il reste à définir un intervalle de temps maximal à la fin duquel la concentration en oxygène doit descendre en dessous de 10 p. 100 pour déclarer que la germination risque d'être affectée. Cet intervalle de temps doit dépendre de la semence, et notamment de la durée de la phase de germination. Le temps nécessaire pour obtenir 50 p. 100 de germination est de 3 jours à $20^{\circ} \mathrm{C}$ et de 7 jours à $10^{\circ} \mathrm{C}$ (PERRY \& HARRISON, 1974 ; RICHARD 1988). Etant donné la faible durée de la phase de germination, on considérera qu'une situation est critique si, partant d'une concentration en oxygène au niveau de la semence de 21 p. 100 , celle-ci s'abaisse à moins de 10 p. 100 en moins de 24 heures. Le choix de cet intervalle de temps maximal reste arbitraire, mais il n'est pas inhérent au modèle et il peut être remis en cause au vu de nouvelles données expérimentales. 


\section{CONCLUSION}

Appliquant les lois physiques de la diffusion, on a défini un système représentatif du fonctionnement du lit de semence en prenant en compte ses caractéristiques spécifiques (croûte de battance), et l'ensemble des paramètres nécessaires pour l'utilisation d'un modèle des transferts gazeux dans le lit de semence en vue de l'étude des conditions d'alimentation en oxygène de la semence de betterave sucrière pendant la germination. Enfin, à partir de données expérimentales ou bibliographiques, on a déterminé les valeurs des paramètres correspondant à des cas-types susceptibles d'être rencontrés dans les conditions du champ cultivé.

Par rapport aux travaux antérieurs, l'utilisation de techniques numériques de résolution des équations différentielles de transfert permettra de calculer l'évolution au cours du temps du profil de concentration en oxygène dans le lit de semence dans n'importe quelle condition, en tenant compte des variations de la fonction puits avec la concentration en oxygène, et en coordonnées tridimensionnelles.

Une des originalités de ce travail est la prise en compte de la distinction entre le volume poral d'origine textural et structural. On a une meilleure caractérisation du volume gazeux où a lieu la diffusion que par la seule porosité libre à l'air, et on peut ainsi relier de façon plus stable des variables d'états du sol à un coefficient de diffusion. Cette liaison est déterminante si l'on veut fournir à terme à l'agronome une grille d'observation du lit de semence et des couches sous-jacentes pour un diagnostic des conditions d'hypoxie des semences.

Certaines limites de l'approche doivent cependant être soulignées :

- on a négligé, comme dans toute la littérature, les transferts par mass-flow. Or, c'est près de la surface du sol, où les gradients et les variations de température et de pression sont les plus forts, que les transferts par mass-flow pourraient être importants à considérer ;

- on a considéré que les variations de la fonction puits à oxygène d'origine microbienne selon la concentration d'oxygène ne dépendaient pas de l'état structural, hydrique et organique du milieu. Or, suivant la taille des éléments structuraux, leurs taux de saturation et la répartition de la matière organique au sein de ces éléments, la fonction puits sera plus ou moins sensible à la concentration en oxygène autour des éléments structuraux. La loi de variation de la fonction puits avec la concentration en oxygène ayant été établie pour des agrégats de petite taille, on risque de surestimer la valeur de la fonction puits et donc de sous-estimer les valeurs de concentration en oxygène dans le lit de semence ;

- enfin, les connaissances actuelles sur la possibilité de germination en liaison avec la consommation d'oxygène ne nous permettent pas de prévoir avec précision le comportement en germination de la semence. On apprécie seulement des niveaux de risques de perturbation de la germination.

Ce modèle, malgré ses limites, va cependant nous permettre d'étudier les conditions d'alimentation en oxygène de la semence de betterave sucrière dans la gamme des situations susceptibles d'être rencontrées dans le champ cultivé en tenant compte de l'anisothermie, de l'anisotropie verticale du profil de sol cultivé et de l'anisotropie horizontale de la croûte. C'est donc un outil précieux de planification d'expériences.

Reçu le $I^{\text {er }}$ décembre 1987. Accepté le 19 avril 1988

\section{RÉFÉRENCES BIBLIOGRAPHIQUES}

Ball B. C., Harris W., Burford J. R., 1981. A laboratory method to measure gas diffusion and flow in soil and other porous materials. $J$. Soil Sci., 32, 323-333.

Boiffin J., 1984. La dégradation siructurale des couches superficielles du sol sous l'action des pluies. Thèse de Docteur Ingénieur. Institut National Agronomique Paris-Grignon. Paris, 320 p.

Boone F. R., Van der Werf H. M. G., Kroesbergen B., Ten Hag F. A., Boers A., 1986. The effect of compaction of the arable layer in sandy soils on the growth of maize silage. 1. Critical matric water potentials in relation to soil aeration and mechanical impedance. Neth. J. agri. Sci., 34, 155-171.

Bouaziz A., 1987. Implantation d'un peuplement de blé tendre en conditions sèches. Analyse physique et modélisation. Thèse de Docteur ès Sciences. Institut Agronomique et Vétérinaire Hassan II. Rabat (Maroc), $101 \mathrm{p}$.

Bridge B. J., Rixon A. J., 1976. Oxygen uptake and respiratory quotient of field soil cores in relation to their air-filled space. $J$. Soil Sci., 27, 279-286.

Brown N. J., Fountaine E. R., Holden M. R., 1965. The oxygen requirement of crop roots and soils under near field conditions. $J$. Agric. Sci., 64, 195-203.

Bruckler L., 1983. Rôle des propriétés physiques du lit de semence sur l'imbibition et la germination. 1. Elaboration d'un modèle du sysième « terre-graine ». Agronomie, 3, 213-222.

Bruckler L., Ball B. C., Renault P., 1988. Laboratory estimation of gas diffusion coefficient and effective porosity in soils. Soil Science (sous presse).

Clifford S. M., Hillel D., 1986. Knudsen diffusion : the effect of small pore size and low gas pressure on gaseous transport in soil. Soil Sci., 141, 289-297.
Currie J. A., 1962. The importance of aeration in providing the right conditions for plant growth. J. Sci. Food Agric., 13, 380-385.

Currie J. A., 1984. Gas diffusion through soil crumbs : the effect of compaction and wetting. $J$. Soil Sci., 35, 1-10.

Dexter A. R., Radke J. K., Hewitt J. S., 1983. Structure of a tilled soil as influenced by tillage, wheat cropping, and rainfall. Soil Sci. Soc. Am. J., 47, 570-575.

Glinski J., Stepniewski W., 1985. Soil aeration and its role for plants CRC Press Boca Raton Florida, 217 p

Grabble A. R., Siemer E. G., 1968. Effects of bulk density, aggregate size, and soil water suction on oxygen diffusion, redox potentials, and elongation of corn roots. Soil Sci. Soc. Am. Proc., 32, 180-186.

Greenwood D. J., 1961. The effect of oxygen concentration on the decomposition of organic materials in soil. Plant Soil, 14, 360-376.

Guérif J., 1986. Modification de la répartition et de l'évolution des matières organiques par la simplification du travail du sol: conséquences sur quelques propriétés physiques, 63-88. In Les rotations céréalières intensives. Dix années d'études concertées INRA-ONIC ITCF 1973-1983, INRA, Paris, 481 p.

Howard P. J. A., Howard D. M., 1979. Respiration of decomposing litter in relation to temperature and moisture. Oikos, 33, 457-465.

Icw Wageningen, 1975. Annual Report «Medeling 153 », 54-55 (en hollandais).

Jaynes D. B., Rogowski A. S., 1983. Applicability of Fick's law to gas diffusion. Soil Sci. Soc. Am. J., 47, 435-430.

Juntilla O., 1974. Effects of low oxygen concentration on the germination of Syringa seeds. Z. Pflanzenphysiol., 74, 168-171. 
Lyda S. D., Robinson G. D., 1969. Soil respiratory activity and organic matter depletion in an arid Nevada soil. Soil Sci. Soc. Am. Proc., 33, 92-94.

Papendick R. I., Runkles J. R., 1965. Transient-state oxygen diffusion in soil : 1 . The case when rate of oxygen consumption is constant. Soil Sci., 100, 251-261.

Parr J. F., Reuszer H. W., 1959. Organic matter decomposition as influenced by oxygen level and method of application to soil. Soil Sci. Soc. Am. Proc., 23, 214-216.

Perry D. A., Harrison J. G., 1974. Studies on the sensitivity of monogerm sugar beet germination to water. Ann. appl. Biol., 77, $51-60$.

Pradet A., Raymond P., 1983. Adenine nucleotide ratios and adenylate energy charge in energy metabolism. Ann. Rev. Plant Physiol., 34, 199-224.

Richard G., Guérif J., 1988. Modélisation des transferts gazeux dans le lit de semence : application au diagnostic des conditions d'hypoxie des semences de betterave sucrière (Beta vulgaris L.) pendant la germination. II. - Résultats des simulations. Agronomie, 8 (7).

Richard G., 1988. La germination des semences de betterave sucrière (Beta vulgaris $L$.) en conditions d'hypoxie : modélisation, contribution $\dot{a}$ un diagnostic au champ. Thèse de Docteur-Ingénieur. Institut National Agronomique Paris-Grignon, Paris, 103 p.

Ross D. J., Cairns A., 1978. Influence of temperature on biochemical processes in some soils from tussock grasslands. 1. Respiratory activity. $N$. Z. J. Sci., 21, 581-589.

Stengel P., 1979. Utilisation de l'analyse des systèmes de porosité pour la caractérisation de l'état physique du sol in situ. Ann. agron., 30, 27-51.

Stengel P., 1986. Simplification du travail du sol en rotation céréalière: conséquences physiques, 15-44. In Les rotations céréalières intensives. Dix années d'études concertées INRA-ONIC-ITCF 19731983. INRA, Paris, 481 p.

Stepniewski W., 1981. Oxygen diffusion and strength as related to soil compaction. 2. Oxygen diffusion coefficient. Pol. J. Soil Sci., 14, 3-13. 